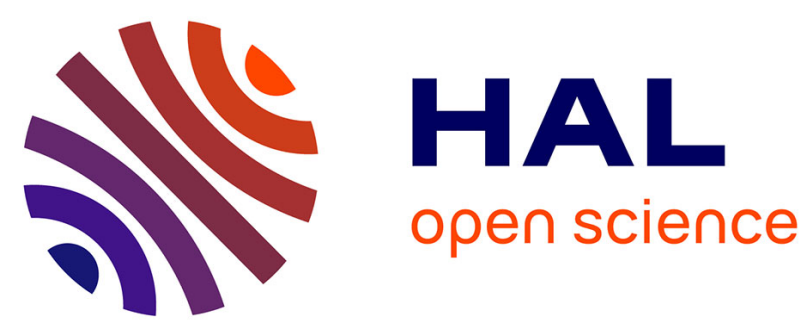

\title{
Thrombin generation in patients with a bleeding tendency of unknown origin
}

Cihan Ay, Johanna Haselböck, Clarissa Laczkovics, Silvia Koder, Ingrid Pabinger

\section{To cite this version:}

Cihan Ay, Johanna Haselböck, Clarissa Laczkovics, Silvia Koder, Ingrid Pabinger. Thrombin generation in patients with a bleeding tendency of unknown origin. Annals of Hematology, 2011, 90 (9), pp.1099-1104. 10.1007/s00277-011-1201-8 . hal-00617078

\section{HAL Id: hal-00617078 \\ https://hal.science/hal-00617078}

Submitted on 26 Aug 2011

HAL is a multi-disciplinary open access archive for the deposit and dissemination of scientific research documents, whether they are published or not. The documents may come from teaching and research institutions in France or abroad, or from public or private research centers.
L'archive ouverte pluridisciplinaire HAL, est destinée au dépôt et à la diffusion de documents scientifiques de niveau recherche, publiés ou non, émanant des établissements d'enseignement et de recherche français ou étrangers, des laboratoires publics ou privés. 
Thrombin generation in patients with a bleeding tendency of

\section{unknown origin}

Cihan Ay *, Johanna Haselböck *, Clarissa Laczkovics *, Silvia Koder *, Ingrid

Pabinger *

* Clinical Division of Haematology and Haemostaseology, Department of Medicine I, Medical University of Vienna, Austria

Correspondence: Prof. Dr. Ingrid Pabinger

Clinical Division of Haematology and Haemostaseology

Department of Medicine I, Medical University of Vienna

Waehringer Guertel 18-20, A-1090 Vienna, Austria

Phone number: +431404004448

Fax number: +431404004030

e-mail: ingrid.pabinger@meduniwien.ac.at

Short title: Thrombin generation and bleeding of unknown origin 


\section{ABSTRACT}

There are a number of persons with a mild to moderate bleeding tendency, in whom no underlying bleeding disorder can be detected despite thorough investigation of all known heritable and acquired haemostatic abnormalities. Thrombin is the central enzyme in the coagulation cascade, which is important for sufficient haemostasis. The measurement of an individual's potential to generate thrombin has been proposed for estimating the individual coagulation potential and predicting a hyper- or hypo-coagulable phenotype. The aim of our study was to investigate in-vivo thrombin generation in a case-control study of patients with a bleeding tendency of unknown origin and in age- and sex-matched healthy individuals. Bleeding tendency was classified according to a standardized bleeding score. Thrombin generation was measured with a commercially available assay (Technothrombin-TGA, Technoclone, Vienna, Austria). In total, 101 patients (76 female, median age $\left[25^{\text {th }}-75^{\text {th }}\right.$ percentile]: 44 [35-60] yrs) and 102 controls (67 women, median age: 47 [38-55] yrs) were enrolled. The distribution of parameters of thrombin generation among patients and controls showed no statistically significant difference: lag phase (14.4 [11.1-18.1] vs. $14.1[12.1-17.1]$ minutes, $p=0.720)$, peak thrombin $(179.8$ [135.6-242.6] vs. 175.1 [143.1-261.4] nM, p=0.576), time to peak thrombin (23.6 [18.1-28.6] vs. 22.6 [18.627.1] minutes, $p=0.790)$, velocity index (19.7 [13.0-39.0] vs. 22.6 [14.5-36.5] $\mathrm{nM} /$ minute, $\mathrm{p}=0.233$ ) and area under the thrombin generation curve (3491 [30693880] vs. 3414 [3045-3750] $\mathrm{nM}$ Thrombin, $\mathrm{p}=0.673)$. In conclusion, the thrombin generation potential in patients with a bleeding tendency of unknown origin was not different from that of healthy individuals.

Keywords: bleeding, thrombin generation, haemostasis, coagulation assay 


\section{INTRODUCTION}

The von Willebrand's disease (vWD) and haemophila are the most frequent and best characterized hereditary disorders that lead to an increased bleeding tendency. Also other rare hereditary abnormalities, such as platelet function defects and deficiencies in fibrinogen, factors II, V, VII, X, XI or XIII, are associated with a moderate to severe bleeding tendency. In some cases an increased bleeding risk may occur as a result of acquired deficiencies in clotting factors or abnormalities of other components of the haemostatic system. However, in clinical practice there are still a number of patients with a mild to moderate bleeding tendency, in whom no underlying bleeding disorder can be detected despite thorough clinical and laboratory investigation of all known hereditary and acquired haemostatic abnormalities. This is especially true for persons who exhibit a bleeding tendency that is solely associated with invasive procedures, in some cases even requiring transfusions. To allow for a targeted therapy of bleeding complications, it is essential to identify the underlying cause. Therefore, the management of bleeding complications in patients with unexplained bleeding tendency is still difficult to tackle.

Thrombin is the central enzyme in the plasmatic coagulation system, which leads to the conversion of fibrinogen to fibrin, a process finally resulting in clot formation and maintaining sufficient haemostasis [1]. Thrombin is also one of the most potent activators of platelets, which are the mainstay of primary haemostasis [2]. The accumulation of thrombin at sites of vascular injury provides an important mechanism for the gathering of platelets and the formation of a growing haemostatic plug. Recent advances in laboratory technologies gave rise to the development of new assays to measure the in-vitro thrombin generation, which reflects the overall coagulation potential [3-6]. 
In contrast to traditional coagulation tests, such as the prothrombin time (PT) and the activated partial thromboplastin time (aPTT), which correspond to artificially created compartments of haemostasis and do not assess the whole coagulation system, the measurement of thrombin generation has been proposed as a very promising approach to globally estimate an individual's coagulation potential and to predict a hyper- or hypo-coagulable state [4-6]. For instance, a high thrombin generation potential has been reported to be associated with an increased risk for thrombotic disorders such as venous thromboembolism [6, 7], while a decreased thrombin generation potential was found in patients at risk of bleeding, such as patients with haemophilia $A$ and $B$, the VWD and other bleeding disorders [8-11]. Data on the thrombin generation potential in patients with a bleeding tendency of unknown origin have been missing so far.

This is why we sought to assess the thrombin generation potential in a casecontrol study of patients with a mild to moderate bleeding tendency of unknown origin compared to age- and sex-matched healthy controls.

\section{METHODS}

\section{Study population}

This case-control study was conducted between 2004 and 2007 at the outpatient clinic of the Department of Medicine I, Clinical Division of Haematology and Haemostaseology of the Medical University of Vienna, Austria.

Patients with a history of bleeding symptoms of unknown origin who had been referred to our outpatient clinic within a period of five years prior to the beginning of the study (years 2000-2004) were identified and contacted personally, by telephone or by postal mail for participation in this study. All patients in whom an inherited or 
acquired haemostatic disorder had been established were excluded. Specific exclusion criteria for the study were: haemophilia $A$ and $B, v W D$, coagulation factor deficiencies and platelet disorders, such as Glanzmann's thromboasthenia or Bernard-Soulier syndrome, or acquired haemostatic deficiencies and defects. Also patients on anti-coagulants, anti-platelet and anti-inflammatory drugs, liver disease (abnormal prothrombin time), renal impairment/insufficiency, thrombocytopenia with a platelet count $<100.000 / \mu \mathrm{l}$, gastro-intestinal (GI) bleeding with a pathologic finding in the Gl-tract and cerebral bleeding due to aneurysm were excluded. Further, subjects with overt infection, pregnancy or malignancy were not included in the study. Eventually, a total of 135 patients with mild to moderate bleeding tendency, in whom all known heritable or acquired bleeding disorders and diseases associated with bleeding risk had been ruled out, were identified to be eligible for the study. The patient population comprised subjects with bleeding conditions associated with trauma, including trauma after surgery or interventions such as dental extraction, a tendency to easy skin bruising, excessive or unprovoked bleeding (e.g. most commonly epistaxis, mucosal and gum bleeding or menorrhagia without a gynaecological/endocrine cause). Of this population 101 patients $(74.8 \%)$ fulfilling the inclusion and exclusion criteria were recruited. At study inclusion, patients underwent a structured interview on their medical and bleeding history.

Hundred-and-two age- and sex-matched healthy individuals without antithrombotic therapy and without a medical history of a bleeding disorder (inherited and acquired bleeding disorders), without a bleeding tendency and without a history of cardiovascular or thrombotic diseases, who were unrelated to the patients, served as controls. The control group was matched to the patient group by sex and age in a whole-group- and not a pair-wise-manner. Individuals in the control group were 
unrelated to each other. They were from the same geographic region and ethnic background as the patients.

The study protocol was approved by the Ethics Committee of the Medical University of Vienna in accordance with the Declaration of Helsinki, and written informed consent was obtained from all study participants prior to any study-related procedures.

\section{Evaluation of bleeding tendency}

To assess the bleeding tendency, a standardized bleeding questionnaire was used, which was already published previously [12]. Each study participant had to answer this structured questionnaire about her/his medical history in order to evaluate bleeding tendency. The questionnaire inquired about 10 present and past bleeding symptoms. The results of the questionnaire were summarized in a specific score as previously published [12]. Specific symptoms included epistaxis, cutaneous symptoms, bleeding from minor wounds, oral cavity bleeding, gastrointestinal bleeding, postpartum haemorrhage, muscle haematomas/haemarthrosis, bleeding after tooth extraction, bleeding after surgery and menorrhagia. The severity of each symptom was subsequently summarized, using a bleeding score system ranging from 0 to 3, as reported in detail previously [12], and considering for each symptom the most severe occurrence. The scale for the entire bleeding score ranged from 0 (minimum) to 30 (maximum).

This structured questionnaire assessing the bleeding tendency was also applied in all controls. All control individuals had a bleeding score of 0 . 


\section{Blood sampling and laboratory analysis}

At study inclusion blood samples were collected by clean and atraumatic venipuncture after overnight fasting using a 21-gauge butterfly needle $(0.8 \times 19 \mathrm{~mm}$; Greiner Bio-One, Kremsmuenster, Austria) for routine laboratory and clotting analysis. The measurement of factor VIII and factor IX was performed according to routine laboratory methods and as described previously [13, 14].

For measurement of thrombin generation venous blood was drawn into a $3.8 \%$ sodium citrate Vacuette ${ }^{\circledR}$ tube (Greiner Bio-One Kremsmuenster, Austria; 9 parts of whole blood and 1 part of sodium citrate stock solution at $0.129 \mathrm{mmol} / \mathrm{L}$ ). Samples were centrifuged to obtain platelet-poor plasma. Aliquots were coded for blind analysis, snap-frozen and stored at $-80^{\circ} \mathrm{C}$ until analyses were performed in series. Thrombin generation was measured with a commercially available assay kit (Technothrombin TGA kit, Techonoclone, Vienna, Austria) on a fully automated, computer-controlled microplate reader (BioTek, FLx800) with a specially adapted software (Technothrombin TGA, Vienna, Austria), using the fluorogenic substrate ZGly-Gly-Arg-AMC (Bachem, Bubendorf, Switzerland) according to the manufacturer's instructions and as previously reported [15]. The concentration of generated thrombin was registered over time, resulting in a thrombin generation curve. From this curve various parameters could be read out describing thrombin activity, such as the lag phase (time until thrombin burst), peak amount of thrombin generation, time to peak thrombin generation, velocity of thrombin generation, or the total amount of thrombin generated (area under the thrombin generation curve, AUC).

\section{Statistical analysis}

Statistical analyses were performed using the Statistical Package for Social Sciences (SPSS version 15.0, SPSS, Chicago, Illinois, USA). Median and 
interquartile range $\left(25^{\text {th }}\right.$ to $75^{\text {th }}$ percentile) of continuous variables are shown. We performed Mann-Whitney $\mathrm{U}$ tests to detect differences in metric variables and in the thrombin generation parameters between patients and healthy controls. Comparison of categorical parameters among the groups was done by the chi-square test. Correlations between metric variables were calculated by the bivariate Spearmantest. A two-tailed p-value of less than 0.05 was considered to indicate statistical significance.

\section{RESULTS}

\section{Patient characteristics}

Table 1 summarizes demographic, clinical and laboratory characteristics of our patients and the age- and sex-matched healthy controls. No statistically significant differences were observed between the two groups with respect to BMI, aPTT, PT, FVIII activity, FIX activity, fibrinogen, and platelet count. No statistically significant difference was present between the patient and the control group with respect to blood group 0 . The median bleeding score in the patient population was 5.0 [range: $1-13]$

\section{Distribution of parameters of thrombin generation in patients and controls}

Statistic analyses showed no significant difference in the distribution of parameters of thrombin generation between patients and controls (Figure 1). The median $\left[25^{\text {th }}-75^{\text {th }}\right.$ percentile] lag phase in patients and controls was 14.4 [11.1-18.1] minutes vs. $14.1[12.1-17.1]$ minutes $(p=0.720)$, velocity index of thrombin generation 19.7 [13.0-39.0] nM/minute vs. 22.6 [14.5-36.5] $\mathrm{nM} /$ minute $(\mathrm{p}=0.233)$, peak thrombin 179.8 [135.6-242.6] nM Thrombin vs. 175.1 [261.4-611] nM Thrombin ( $p=0.576)$, time 
to peak $23.6[18.1-28.6]$ minutes vs. $22.6[18.6-27.1]$ minutes $(p=0.790)$ and the AUC 3496 [3069-3880] nM thrombin vs. 3314 [3045-3750] nM thrombin $(p=0.673)$. Also in separate analyses for women and men no difference in thrombin generation was found between patients and controls (data not shown).

\section{Correlation of thrombin generation with clinical and laboratory parameters}

We evaluated the correlation coefficients between key variables of thrombin generation in the patient group and compared them with the corresponding coefficients in the control group. As the results were broadly similar, the correlation coefficients for the entire study population are presented in Table 2. No relevant correlations were observed between age, BMI, PT, FIX activity or fibrinogen and the lag phase, peak thrombin and AUC. A weak but statistically significant correlation of the lag phase, peak thrombin and AUC was present with aPTT and FVIII activity.

\section{DISCUSSION}

Management of a patient with bleeding can be quite challenging in clinical practice, but accurate diagnosis of the cause of bleeding is crucial for devising appropriate therapeutic strategies. Thorough clinical assessment remains the cornerstone in the diagnostic algorithm for bleeding disorders, including medical history, family history, use of medications and physical examination, followed by global tests of haemostasis to screen patients clinically suspected of having a bleeding disorder and to identify the part of the haemostatic system that is aberrant for further diagnostic work-up and for getting deeper insight into the underlying pathogenetic mechanism [16]. Recently, thrombin generation tests have been proposed to assess global haemostasis and its disorders [4]. Moreover, thrombin is a 
key factor in haemostasis, and the measurement of thrombin generation has been applied for investigating the risk of bleeding in patients with a bleeding disorder $[6,8-$ 11].

In the present case-control study we therefore assessed the in-vitro thrombin generation potential in patients with a mild to moderate bleeding tendency in whom no underlying cause could be identified despite comprehensive routine clinical and laboratory investigations, comparing it to age- and sex-matched control individuals without a bleeding or thrombotic disease. We found that parameters measured by the thrombin generation assay in patients did not differ from those measured in controls. In support of this finding, also traditional coagulation tests exploring the coagulation system, such as the aPTT and the PT, were equally distributed in both groups. Furthermore, median levels of the FVIII and FIX activity, fibrinogen and platelet count were comparable between patients and the control population.

From the clinical point of view there is a great interest in having tests that assess overall haemostasis and the coagulation potential for screening, diagnosis of a bleeding disorder and evaluation of the individual clinical phenotype of patients with a bleeding tendency. It was reported that parameters of the thrombin generation assay correlate with bleeding tendency in haemophila or rare inherited bleeding disorders [8, 11], however, the correlation with severity and the clinical phenotype of the bleeding disorder is still relatively poor. Previous studies have shown a marked heterogeneity in thrombin generation at similar FVIII levels (reviewed in [6]) and test results seem to be more sensitive to very low levels of coagulation factors [11]. In this context, it has to be pointed out that in our study only patients with a mild to moderate bleeding tendency were included and the presence of severe haemostatic deficiencies was unlikely. Interestingly, in some coagulation disorders, such as FXI, FVII or FXIII deficiency, the relationship between the level of factor deficiency and the 
clinical phenotype and severity of bleeding tendency is less clear [16]. Another aspect of our study is that symptoms suggestive of a mild bleeding tendency are common in the general population. Although such symptoms are often associated with an underlying bleeding disorder, they may not always reflect the presence of a disorder of the haemostatic system. To evaluate the bleeding tendency, all of our study participants had to answer a standardized and previously published bleeding questionnaire [12]. The median bleeding score in patients was 5, which was indicative of a mild/moderate bleeding tendency. As expected, all controls had a bleeding score of 0 .

Until now, several assays have been developed to measure the in-vitro thrombin generation in plasma. In our study, we used a commercially available assay that has been broadly applied in previously published studies (reviewed in [5]). However, the use of thrombin generation tests as a tool for identifying a hypocoagulable state and for evaluating the bleeding risk and clinical phenotype in patients with coagulation disorders is still not established in routine practice, because these tests are not yet standardized with respect to preanalytical and analytical conditions [3, 17-19]. Of particular importance in order to obtain reproducible and meaningful results are adequate sampling conditions, manipulation of plasma samples and performing measurements under standardized conditions. We treated samples of patients and controls in exactly the same way, and measurement of thrombin generation was done by the same person in all study participants under the same conditions.

The main limitations of our study are the relatively small number of study participants and the retrospective case-control design. However, we performed additional coagulation analyses and correlated these results to thrombin generation parameters in patients and controls. As expected, thrombin generation parameters 
showed a weak, but statistically significant correlation with the aPTT and the FVIII activity. FVIII is considered one of the important determinants of thrombin generation [6]. As patients were measured not during the acute phase of the bleeding symptoms pertinent to their bleeding tendency, but a certain time thereafter, we cannot exclude that thrombin generation might have been disturbed during the acute phase of bleeding.

In summary, parameters of the thrombin generation potential did not differ between patients with a bleeding tendency of unknown origin and healthy controls, which suggests that the thrombin generation potential in patients with a bleeding tendency of unknown origin was not disturbed. Similarly to the traditional coagulation tests, the thrombin generation assay did not provide additional information to explain bleeding in these patients.

Legend to Figure 1: Box plot analyses of the distribution of thrombin generation parameters (A: lag phase, B: velocity index, C: peak thrombin, D: time to peak, E: AUC) in patients with a bleeding tendency of unknown origin and healthy controls. The difference in the median levels of thrombin generation parameters between patients and controls was not statistically significant. 


\section{ACKNOWLEDGEMENTS}

We thank Tanja Altreiter, MA (Clinical Division of Haematology and Haemostaseology, Department of Medicine I, Medical University of Vienna) for proofreading this manuscript.

Furthermore, we are thankful to Technoclone (the late Professor Bernd Binder and Mrs. Veronika Binder) for the donation of the Technothrombin TGA assay kits and for their assistance.

\section{Funding}

The study was funded by an unrestricted grant from CSL Behring Austria. 


\section{REFERENCES}

1. Crawley JT, Zanardelli S, Chion CK, et al (2007) The central role of thrombin in hemostasis. J Thromb Haemost Suppl 1:95-101.

2. Brass LF (2003) Thrombin and platelet activation. Chest 124(3 Suppl):18S25S.

3. Kitchen S, Hayward C, Negrier C, et al (2010) New developments in laboratory diagnosis and monitoring. Haemophilia Suppl 5:61-6.

4. Nair SC, Dargaud Y, Chitlur M, et al (2010) Tests of global haemostasis and their applications in bleeding disorders. Haemophilia Suppl 5:85-92.

5. Baglin $\mathrm{T}(2005)$ The measurement and application of thrombin generation. $\mathrm{Br}$ J Haematol 130:653-61.

6. van Veen JJ, Gatt A, Makris M (2008) Thrombin generation testing in routine clinical practice: are we there yet? $\mathrm{Br} \mathrm{J}$ Haematol 142:889-903.

7. Pabinger I, Ay C (2009) Biomarkers and venous thromboembolism. Arterioscler Thromb Vasc Biol 29:332-6.

8. Dargaud $\mathrm{Y}$, Béguin S, Lienhart A, et al (2005) Evaluation of thrombin generating capacity in plasma from patients with haemophilia A and B. Thromb Haemost 93:475-80.

9. Rugeri L, Beguin S, Hemker C, et al (2007) Thrombin-generating capacity in patients with von Willebrand's disease. Haematologica 92:1639-46.

10. Rugeri L, Quélin F, Chatard B, et al (2010) Thrombin generation in patients with factor XI deficiency and clinical bleeding risk. Haemophilia 16:771-7.

11. Al Dieri R, Peyvandi F, Santagostino E, et al (2002) The thrombogram in rare inherited coagulation disorders: its relation to clinical bleeding. Thromb Haemost 88:576-82. 
12. Rodeghiero F, Castaman G, Tosetto A, et al (2005) The discriminant power of bleeding history for the diagnosis of type 1 von Willebrand disease: an international, multicenter study. J Thromb Haemost 3:2619-26.

13. Ay C, Thom K, Abu-Hamdeh F, et al (2010) Determinants of factor VIII plasma levels in carriers of haemophilia A and in control women. Haemophilia 16:111-7.

14. Vormittag R, Bencur P, Ay C, et al (2007) Low-density lipoprotein receptorrelated protein 1 polymorphism $663 \mathrm{C}>\mathrm{T}$ affects clotting factor VIII activity and increases the risk of venous thromboembolism. J Thromb Haemost 5:497-502.

15. Ay L, Kopp HP, Brix JM, et al (2010) Thrombin generation in morbid obesity: significant reduction after weight loss. J Thromb Haemost 8:759-65.

16. Greaves M, Watson HG (2007) Approach to the diagnosis and management of mild bleeding disorders. J Thromb Haemost Suppl 1:167-74.

17. Dargaud $Y$, Luddington R, Gray E, et al (2010) Standardisation of thrombin generation test--which reference plasma for TGT? An international multicentre study. Thromb Res 125:353-6.

18. Dargaud Y, Luddington R, Gray E, et al (2007) Effect of standardization and normalization on imprecision of calibrated automated thrombography: an international multicentre study. $\mathrm{Br} \mathrm{J}$ Haematol 139:303-9.

19. Dargaud Y, Negrier C (2010) Thrombin generation testing in haemophilia comprehensive care centres. Haemophilia 16:223-30. 
Figure 1
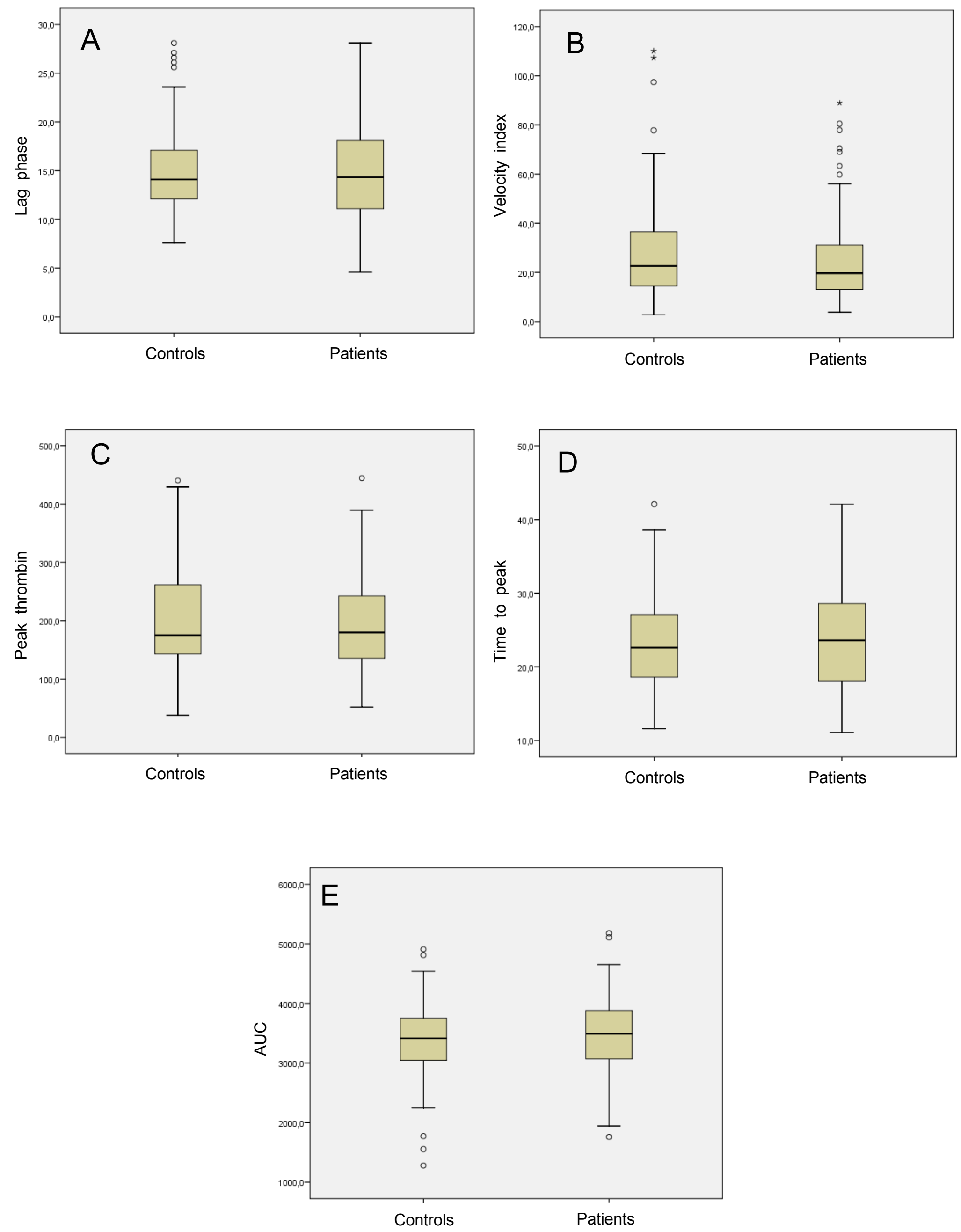
Table 1. Demographic, clinical and laboratory results in patients with a bleeding tendency of unknown origin compared to healthy controls.

\begin{tabular}{|l|l|l|l|}
\hline & \multicolumn{1}{|c|}{ Patients $(\mathrm{n}=101)$} & \multicolumn{1}{|c|}{ Controls $(\mathrm{n}=102)$} & $p$-value \\
\hline Women, $\mathrm{n}(\%)$ & $76(75 \%)$ & $67(66 \%)$ & \\
\hline Blood group O, $\mathrm{n}(\%)$ & $39(39 \%)$ & $44(43 \%)$ & 0.136 \\
\hline & Median [25th-75th percentile] $]$ & Median [25th-75th percentile] & \\
\hline Age at study entry, & $44[35-60]$ & $47[38-55]$ & 0.939 \\
\hline years & & & 0.773 \\
\hline BMI, kg/m ${ }^{2}$ & $23.8[21.5-26.5]$ & $24.0[21.5-26.4]$ & 0.361 \\
\hline aPTT, sec & $35.5[33.5-38.8]$ & $35.5[33.3-37.4]$ & 0.621 \\
\hline Prothrombin time, \% & $96[89-103]$ & $96[92-102]$ & 0.976 \\
\hline FVIII activity, \% & $136[111-164]$ & $137[111-164]$ & 0.742 \\
\hline FIX activity, \% & $109[92-122]$ & $105[91-120]$ & 0.070 \\
\hline Fibrinogen, mg/dL & $322[287-356]$ & $311[260-343]$ & 0.257 \\
\hline Platelet count, G/l & $248[211-279]$ & $249[221-286]$ & \\
\hline
\end{tabular}

Abbreviations: BMI, Body mass index; aPTT, activated partial thromboplastin time; FVIII, factor VIII; FIX, factor IX 
Table 2. Spearman correlation coefficient $\left(r^{*}\right)$ of lag phase, peak thrombin and AUC with age, BMI, aPTT, prothrombin time, FVIII, FIX, fibrinogen, and platelet count.

\begin{tabular}{|l|ll|ll|ll|}
\hline & \multicolumn{2}{|c|}{ Lag phase } & \multicolumn{2}{c|}{ peak thrombin } & \multicolumn{2}{c|}{ AUC } \\
& $\mathrm{r}$ & $\mathrm{p}$-value & $\mathrm{r}$ & $\mathrm{p}$-value & $\mathrm{r}$ & $\mathrm{p}$-value \\
\hline Age & 0.035 & 0.625 & 0.068 & 0.339 & 0,069 & 0.328 \\
\hline BMI & 0.093 & 0.204 & 0.082 & 0.263 & 0.070 & 0.341 \\
\hline aPTT & 0.233 & 0.001 & -0.226 & 0.001 & -0.219 & 0.002 \\
\hline Prothrombin time & -0.140 & 0.048 & 0.125 & 0.076 & 0.137 & 0.052 \\
\hline FVIII activity & -0.163 & 0.021 & 0.322 & $<0.001$ & 0.276 & $<0.001$ \\
\hline FIX activity & -0.005 & 0.943 & 0.138 & 0.052 & 0.119 & 0,092 \\
\hline Fibrinogen & 0.142 & 0.045 & 0.073 & 0.301 & 0.086 & 0.222 \\
\hline Platelet count & -0.132 & 0.063 & 0.156 & 0.027 & 0.221 & 0.002 \\
\hline
\end{tabular}

